\title{
Integrated Query Data Communication using Arti-Q System for Placement Cell Automation
}

\author{
Karthika S, Marshia Theras S, Mukesh K
}

\begin{abstract}
Maintaining database of students in colleges is being an issue for placement cell since the data is maintained online and it is difficult to get access the system. Hence come up with idea of maintaining a database in offline method using a integrated ARTI-Q system which is using simple tools like Microsoft visual studio(version 2008) as front end and Microsoft SQL server(version 2005) as back end. The main aim of this project is to move packets from source to destination without an internet connection. Packets will only communicate through the assistance of base stat ions in which they are transmitted as Unicode transmission. The high transmission baud rate 115200 is set to increase the transmission. The program must aim to consolidate all divisions and roles across a college into a centralized database that can meet specific needs of all those departments. The information about campus interview will be sent to the placement officer and he will put information about the drive in the server. The system will check criteria from database for the list of individuals meeting the company criteria and the information will be sent to non-placed students.
\end{abstract}

Keywords---.NET, database, SQL server, messages, query.

\section{INTRODUCTION}

The placement cell uses a online server to maintain database of personal details as well as academic details in its server and to access the details the device needs to be connected to a internet connection which is not possible in all the areas hence we have designed a offline base database server which will be in a centralized manner so every legal user can have access to it, the system will use Microsoft visual studio for front end and microsoft SQL server as its back end. The user will have to send a request to the central device to access the information and after verification the data could be accessed by the user. Also the information about campus interview will be sent to the placement officer and he will put information about the drive in the server. The system will check criteria from database for the list of individuals meeting the company criteria and then the information will be sent to non- placed students.

Revised Manuscript Received on February 05, 2020.

* Correspondence Author

Karthika S*, Department of Electronics and Communication Engineering, Sri Krishna College of Technology (SKCT), Coimbatore, Tamil Nadu, India Email:karthika.sivakumar9449@gmail.com

Marshia Theras S, Department of Electronics and Communication Engineering, Sri Krishna College of Technology (SKCT), Coimbatore, Tamil Nadu, India Email:s.marshiatheras@gmail.com

Mukesh K, Department of Electronics and Communication Engineering, Sri Krishna College of Technology (SKCT),Coimbatore, Tamil Nadu, India Email:mukeshhrithik66@gmail.com

(C) The Authors. Published by Blue Eyes Intelligence Engineering and Sciences Publication (BEIESP). This is an open access article under the CC BY-NC-ND license (http://creativecommons.org/licenses/by-nc-nd/4.0/)

\section{LITERATURE SURVEY}

[1] Codetracker,A lightweight approach to tracking SMS auth orisation codes and protecting them. Specifically, use of taint tracking to mark the authorization code at the origin of incoming SMS messages (taint sources) with taint tags and then scatter the system tags.

[2] Highly secured ARTI-Q based binary encoded data swapping and transmission system is a term coined to eradicate the human error by super sending the manual system. This kit affects the powerful performance of real time application software based on the commercial enterprise. [3]During data network congestion the use of SMS to transfer small data packets. This will keep key mobile applications for data transfer even during periods of high workload or unavailability of the mobile network.

[4] In the current state of the art mobile phones use ad-hoc networks. It enables wireless communication and the mobile users roam from cell to cell. The central location switching center is responsible for the data communication in this network.

[5] Packet transmission requires waiting for process completion, this is critical to the packet / data loss experience in a congested network. Network congestion is tangential to processing speed, transmission rate, bandwidth, type of switching mechanism (router or hub), transmission medium,

number of arrival packets vs. number of packets processed and number of departure packets. It ensures that if packet arrival exceeds packet departure, network congestion is expected to result in packet drop and packet loss, and if packet arrival. There must be no network congestion, therefore no packet drop and no data / packet loss, less than packet departure.

[6] Result Alert System is a system that can be used to address the challenges that most of our higher learning institutions face when giving students. SMS and e-mail play an important part in implementing this system SMS and email today have become valuable tools because of their utility in transmitting knowledge from one position to the next very quickly and easily.

[7] The system called Sulula, provides an infrastructure for distributing private data in challenged networks. A typical use pattern for this program involves creating or aggregating data at the source,

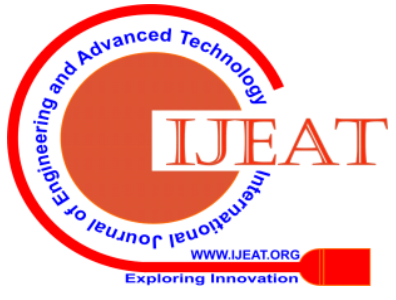




\section{Integrated Query Data Communication using Arti-Q System for Placement Cell Automation}

requesting data from the user, providing data through the

Sulula network and finally data consumption.

Some drawbacks are identified from the above references. Wireless bandwidth and client's battery power are the two major resources. To make an efficient system, first thing is measuring these resources Wide variations and rapid changes in network conditions and local resource availability have far fewer computing power and memory capacity when accessing remote data Due to low bandwidth in the wireless environment there is a problem occurs from the server side. Reason of congested network the packet transmission will be delayed and also reduce the system speed. Using different programming languages such as PHP,CSS are having lot of functions to compile and run ,so that the execution time whole program is delayed. The disadvantage of all the above program is to the tempering of data, increase the speed of transmission and provide protection that protects against outsider crime.

\section{PROPOSED SYSTEM}

It is being developed as a replacement for the existing system, primarily an application oriented which could be enabled in the future. To overcome the placement drawbacks, the entire system is improved in order to boost the management side by automatically sensing the management queries and response to the queries. The Query for the management is as follows RNA (Retrieve Student Academic details), RNP (Retrieve Student personal Details), RPD (Retrieve Placement details), Placed (Retrieve Placed student details). To build a user friendly program that meets the user's needs whenever needed .Information can be created and altered by admin.

Some advantages of the proposed system include: Increases the user satisfaction, Data tampering is reduced, Provides security that protect against illegal user and reduces cost of operations.

This management system consists a collection of interrelated data and packets of programs to access the given data. It is the database, which contains information about a specific entry. DBMS ' main objective is to provide an infrastructure that is easy and efficient to use for data base

retrieval and storage. A principal aim of the database system is to provide a limited view of the data to users. A database contains two language types. They are: Database Definition Language, used to specify the database scheme and the Data Manipulation Language is used to show database queries and updates 


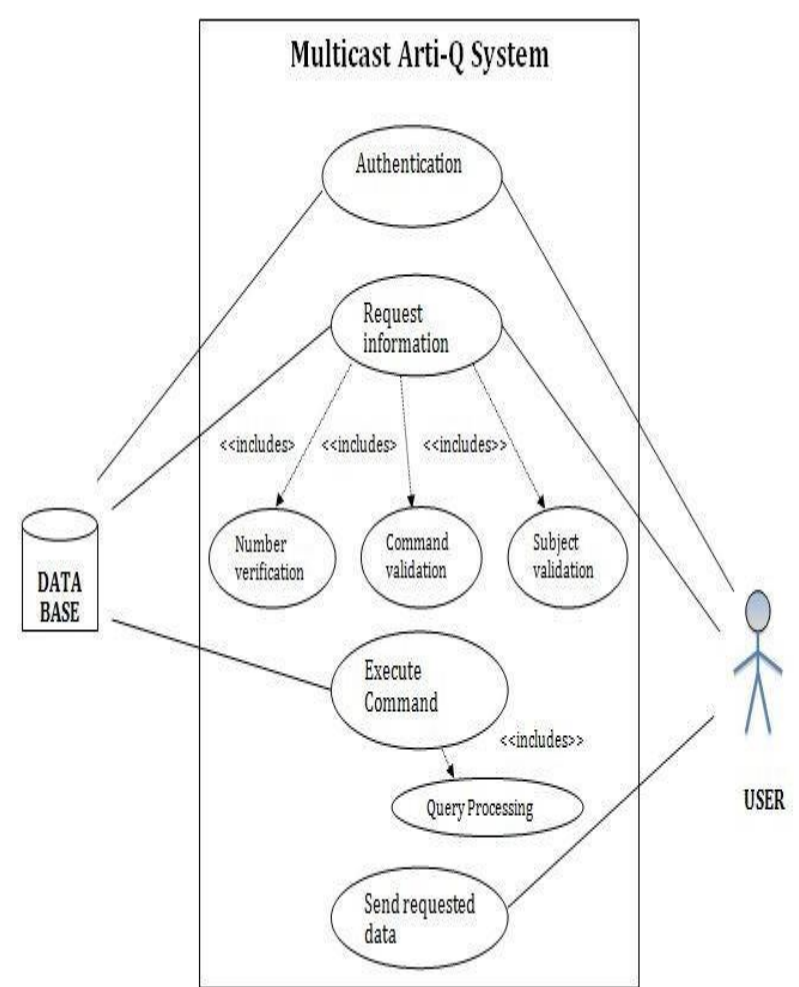

Figure 3.2: Interface between user and data base.

The interface architecture shows how the device communicates with both the user and the modules. The code tells the system what action to take to enter, modify or retrieve data. System design is the solution, including the understanding and organizational details needed to implement the proposed system in a feasibility study, on how to approach create a new structure. The goal of proposed system is to explain the driving forces behind the design process which will lead to the system's final design.

The main phase system in the design process is input design. It is the process where the obtained feedback is organized and configured to extract from the user the necessary information, removing the unnecessary information. The goal of input design is to provide the highest level of accuracy possible and to ensure that the user easily interprets the data.

Proper security is maintained both in the user level and in the level of management. The data is stored as RDBMS in Access 2000, which is highly reliable and user- friendly, and user-level security is managed using password options and sessions to ensure all access is secure.

\section{RESULT AND DISCUSSION}

The statistics below indicate the user must provide passwords to gain access to the system .Then the details required by the user can be selected from the available data. The details may also be updated if necessary and the required or updated database will finally be stored in a table format that will be use $d$ as required.

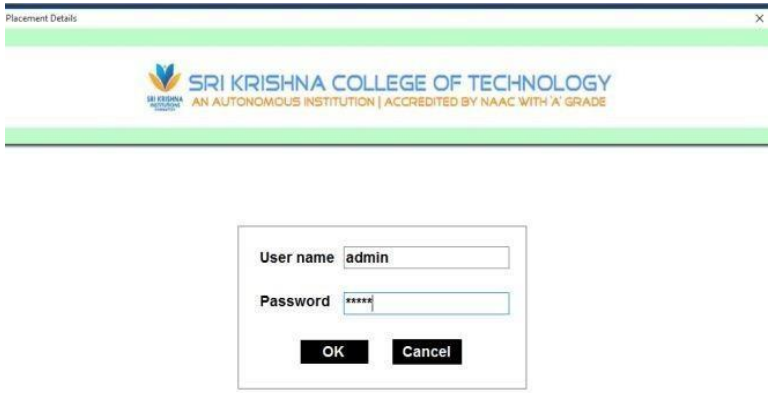

Figure 4.1: Log in page.

First the admin credentials are obtained. Log in page is used to enroll user's confidential page.

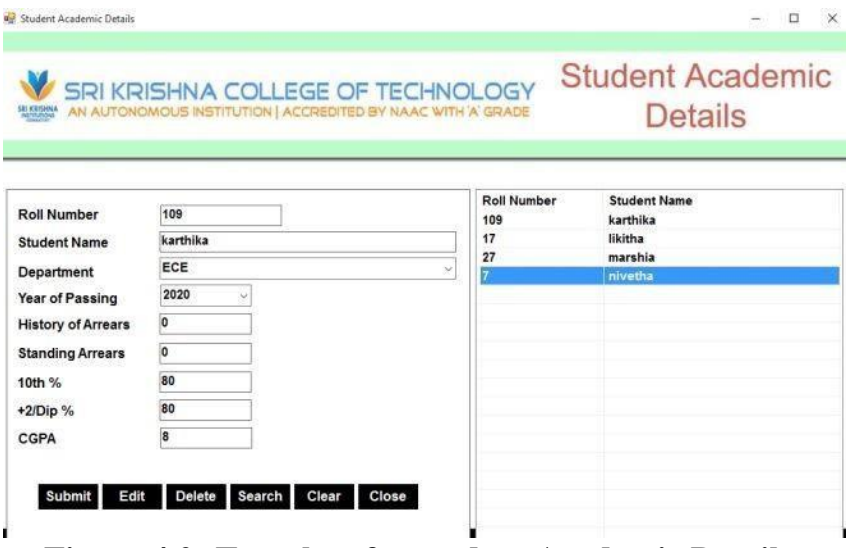

Figure 4.2: Template for student Academic Details.

The details present in the template will be like a application form. Based on the user requirements we can change the template.

The system's main factor is the performance. Performance design is the process involving the design of the necessary outputs that will assist the user according to their needs. The detailed design of the output should enhance the device relationship with the customer and help with future decisions.

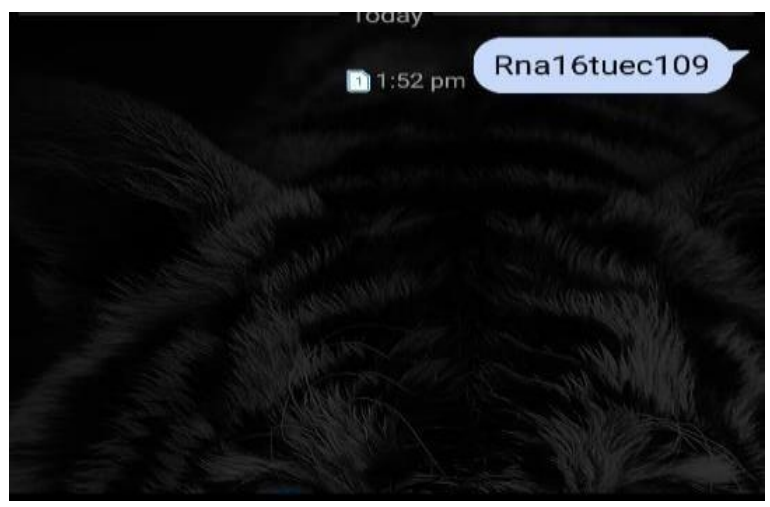

Figure 4.3: Requesting the academic details of a student.

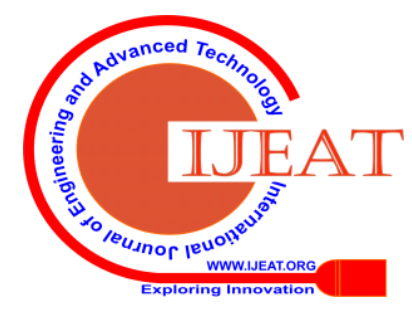




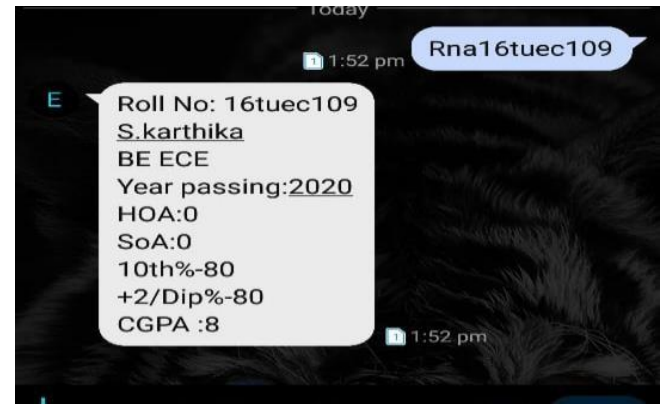

Figure 4.4 : Receiving academic knowledge from SQL server.

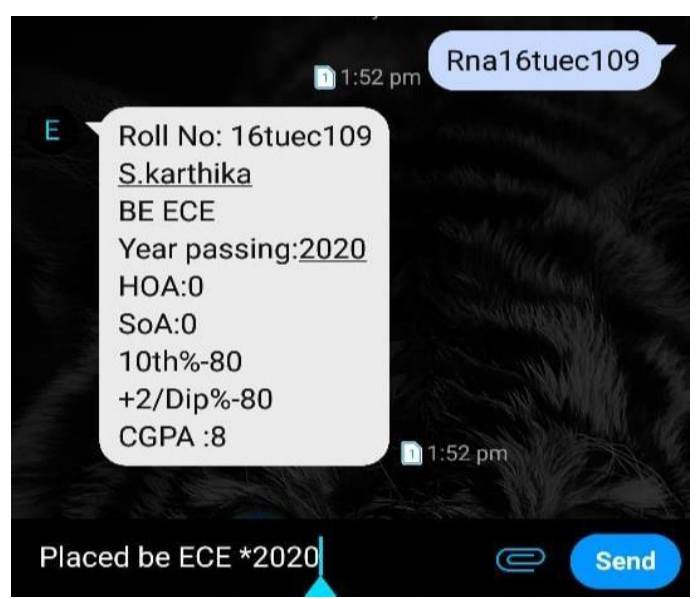

Figure 4.5 : Requesting placed students details.

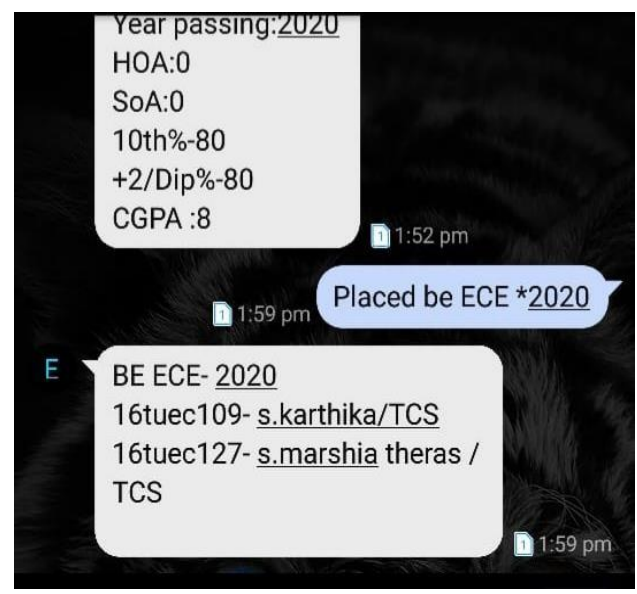

Figure 4.6 : Receiving placed student knowledge from the SQL server.

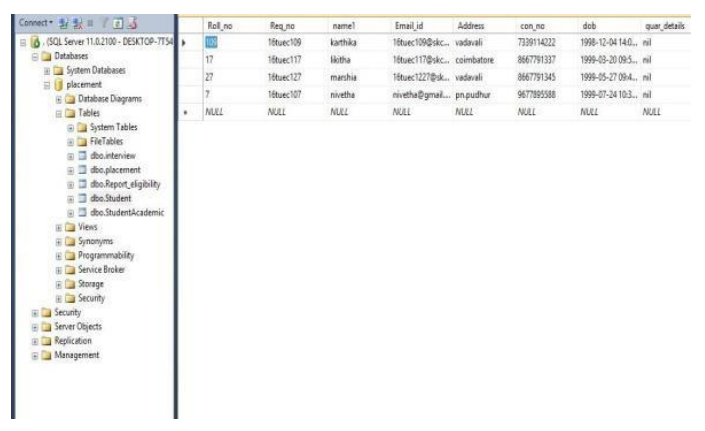

Figure 4.7: Stored data in server.
After updating the details into the database the sql server will store the data in the table format.

Table 4.8: Comparitive table.

\begin{tabular}{|c|c|c|}
\hline & EXISTING & PROPOSED \\
PARAMETERS & SYSTEM & SYSTEM \\
\hline $\begin{array}{c}\text { Bandwidth(baud } \\
\text { rate) }\end{array}$ & 9600 & 9600 to115200 \\
\hline Language Used & PHP & .Net, SQL \\
\hline Device & Mobile phone & GSM module \\
\hline
\end{tabular}

\section{CONCLUSION}

This project is implemented to replace the manual system effectively. The project works on windows technology, The system can be uploaded into a company to make it available globally and secured. The software (ASP.Net, VB.Net and ADO.Net) used to develop the system makes it more flexible, portable more secure. It also supports dynamic content forms. The project is full-fledged and user- friendly. The system reduced the clerical overhead considerably and drastically reduced the time taken in the products. The device meets all the user requirements. To my experience I conclude the program as best as possible.

\section{REFERENCE}

1. Jinku Li, Yangtian Ye, Yajin Zhou, Jianfeng Ma,"Code Tracker: A Lightweight Way to Track and Secure Authorization Codes in SMS Messages. [2]S.Deepa, Dr. D.Francis Xavier Christopher,"Highly Secured Based ARTI- Q Binary Encoded Data Swapping and Transmission System" in the course of Internat Research in Engineering Applications \& Management,2019.

2. Hayala N. Curto, Humberto Torres MarquesNeto, Artur Ziviani, Jussara

3. M. Almeida, Josemar A. Caetano, Carlos Henrique S.Malab,"Brazilian Symposium on Computers and Distributed Systems "2015: Using SMS during the Heavy Workload Periods to transfer small data packets to mobile data networks..

4. Mr. Nitin V. Wankhade, Dr. S.P.Deshpande,"Ad-hoc Network Data Comm unication: Problems and Issues" in the proceedings of the International Journal of Advanced Research in Computer and Communication Engineering,2013. [5]Promise T. K. Akiene, Ledisi G. Kabar "Optimizing Data Packet Transmiss

5. ion in a Congested Network ,"International Journal of Computer Networks and Security,2015.

6. In proceedings with IOSR Journal of Mobile Computing \& Authentication (IOSRJ-MCA), Olusanya Olamide, Onazi Ogaba and O, "An SMS and e- mail response alert system."

7. "Distribution of private data in threatened network environments,"in the proceedings of Azarias Reda, Brian Noble and Yidnekachew Haile at the 19th International World Wide Web Conference. (2010) End quote: (2010) [8]"Data mining security- A comprehensive survey. Global Computer

8. Science and Technology Journal: Niranjan A. Software \& Data Engineering (2016) (English version).

9. "Data Mining Safety," with Data Mining Issues of Importance-A Survey. N amrata Chandrakar \& Shreya Jain Rajabhoj's Imperial Journal of Interdiscipliary Research (IJIR), (2017).

10. Muhammad Jawad Mughal Hamid. (1998). Web Data Mining Methods, Software and Algorithms: An Overview.(IJACSA) International Journal of Advanced Computer Science and Applications. 